\title{
Bill Sandholm in Memoriam
}

\section{Larry Samuelson $^{1} \cdot$ Jörgen Weibull ${ }^{2}$}

Published online: 11 September 2020

(c) Springer-Verlag GmbH Germany, part of Springer Nature 2020

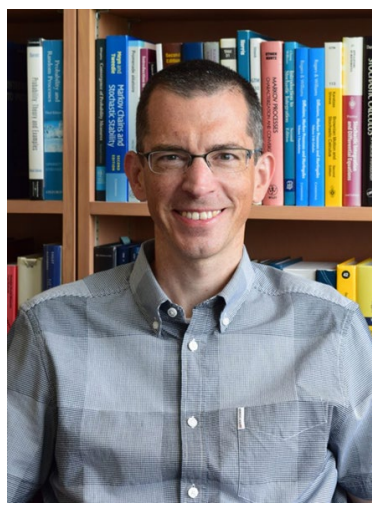

William H. Sandholm, 1970-2020

As were so many others, we were very saddened to hear that William "Bill" Sandholm passed away on July 6, 2020, at the all-too-young age of 49. Bill was an excellent and insightful researcher, an inspiring colleague, and a trusted friend.

Bill graduated from Dartmouth College in 1992, with a major in Economics, and received his Ph.D. from Northwestern's Kellogg Graduate School of Economics in 1998. He joined the Department of Economics at the University of Wisconsin that fall, and spent the next 22 years at Wisconsin, rising to become the Richard E. Stockwell Professor of Economics. In the process, he became a fixture in the economic theory and game theory communities. He served on the editorial boards of Dynamic Games and Applications, Games and Economic Behavior, the International Journal of Game Theory, the Journal of Dynamics and Games, the Journal of Economic Behavior and Organization, the Journal of Economic Theory, Mathematics

Jörgen Weibull

jorgen.weibull@hhs.se

1 Yale University, New Haven, CT, USA

2 Stockholm School of Economics, Stockholm, Sweden 
of Operations Research, and Theoretical Economics. He served on several National Science Foundation panels. He was a member of the Council of the Game Theory Society. He was tireless as an unselfish meeting organizer and referee.

Bill had a wide range of interests-he was as interested in Dostoyevsky as in Dedekind, and as comfortable with Cartesian philosophy as with Cartesian coordinates-but his focus was on evolutionary game theory. Bill pursued his interest in evolutionary game theory with a wide range of coauthors, to whom we apologize for not mentioning in the text, trusting readers to refer to the following bibliography.

Three main themes appear in Bill's research: (1) the application of evolutionary game theory to implementation problems in economics, (2) the convergence of behavior in evolutionary game theory, and (3) the micro foundations of evolutionary dynamics. On the first theme Bill was a pioneer. In a series of papers ("Evolutionary Implementation and Congestion Pricing" (Review of Economic Studies, 2002), "Negative Externalities and Evolutionary Implementation" (Review of Economic Studies, 2005), and "Pigouvian Pricing and Stochastic Evolutionary Implementation" (Journal of Economic Theory, 2007), Bill provided a general framework demonstrating how a benevolent government can use simple pricing schemes to ensure efficient behavior in implementation problems with hidden information and hidden actions. The key to this analysis is the notion of evolutionary implementation, invented by Bill for this purpose. Evolutionary implementation is a strong form of the requirement that players who follow a reasonable and quite general myopic adjustment process eventually learn to behave as the planner desires. In "Evolutionary Implementation...," Bill solves a network planner's problem, showing that efficient behavior can be evolutionarily implemented using a simple, decentralized price scheme. (More precisely, he constructs a price scheme under which the set of efficient outcomes is globally stable under any reasonable evolutionary process.) In "Negative Externalities...", Bill extends these results to a general model of negative externalities, as occurs whenever (for example) my decision to use a highway imposes a cost on you via increased congestion. In "Pigouvian Pricing...", Bill obtains yet more general implementation results for a model in which externalities may be negative, positive, or some combination of the two. The pricing scheme he employs defines a game which may possess multiple equilibria, but he exploits his previous theoretical results to isolate a unique and efficient outcome.

The key to evolutionary implementation is the convergence of behavior. It is then no surprise that Bill was interested in the convergence properties of evolutionary dynamics, the second main theme in his research. In two papers, "Potential Games with Continuous Player Sets" (Journal of Economic Theory, 2001) and "Excess Payoff Dynamics and Other Well-Behaved Evolutionary Dynamics" (Journal of Economic Theory, 2005), Bill introduces general classes of population games which both have desirable convergence properties and which are relevant in economic applications. In the first paper, he shows that in potential games, for dynamics which satisfy a weak characteristic (payoff monotonicity) of coherent behavior, convergence occurs to equilibrium play. In "Excess Payoff Dynamics...", Bill introduces stable games, a more general class of games that includes many standard games as special cases. He shows that while payoff monotonicity of an evolutionary dynamic is insufficient to guarantee convergence to equilibrium in these games, 
supplementing this requirement with some mild technical conditions ensures that convergence occurs. A third paper, "On the Global Convergence of Stochastic Fictitious Play" (Econometrica, 2002) addresses a problem in fictitious play that first attracted the attention of economists and mathematicians in the 1960s, providing some of the strongest convergence results yet found. In "The Projection Dynamic and the Replicator Dynamic" (Games and Economic Behavior 2008), "The Projection Dynamic and the Geometry of Population Games" (Games and Economic Behavior 2008) and "Local Stability under Evolutionary Game Dynamics" (Theoretical Economics, 2010), Bill very nicely extends his results to new classes of dynamics. Together, these papers provide what are now the standard tools for studying convergence in evolutionary models.

At the same time, Bill was careful to identify the limits of his work. It is intuitively evident that some conditions are needed to ensure the convergence of an evolutionary system. However, it seems just as intuitive that evolutionary dynamics should quite generally purge strictly dominated strategies. Bill's 2011 Theoretical Economics paper shows that this need not be the case. The key to his example is a pure strategy that is strictly dominated by a mixture of three pure strategies. The population shares of these three strategies cycle perpetually over time, precluding convergence to the dominating mixed strategy, thereby allowing the strictly dominated pure strategy to survive.

The initial work in evolutionary game theory focused on models of random matching, in which members of a population are repeatedly matching into pairs to play an underlying game. This model grew naturally out of interest in using evolutionary arguments to evaluate equilibria in games, and has the advantage of endowing payoffs with a linear structure. Bill was among the first to extend this reasoning to more general population games, in which individual payoffs can be quite general functions of the population strategy profile, as, for example, in networks. Perhaps more important than the increased generality per se, this approach allowed Bill to simplify and identify the essence of the central arguments. This approach culminated in his 2010 MIT Press book, Population Games and Evolutionary Dynamics. A notable aspect of the reaction to this book, now a standard reference in this area, is that it is routinely characterized simply as "beautiful."

While evolutionary population models commonly take the form of differential equations characterizing the aggregate behavior of a population, this aggregate behavior presumably reflects an underlying vision of the individual. As the third main theme in Bill's research, he investigated the behavioral foundations of evolutionary dynamics, examining Markov processes based on specifications of individual behavior. The formulation of such models is relatively straightforward, but there is an art to extracting useful results. In "Evolution and Equilibrium under Inexact Information" (Games and Economic Behavior, 2003), Bill uses approximation results from probability theory to show that under broad assumptions, aggregate behavior in such models follows an almost deterministic trajectory (given by the solution to an ordinary differential equation derived from the expected motion of the process) for a very long time. This result provides a useful description of disequilibrium adjustments in individual behavior while retaining much of the convenience of the population-level approach. However, in some circumstances it reveals little 
about the sorts of behaviors that emerge in the long run, in what is referred to as the steady state equilibrium of the model. Bill therefore identifies another stochastic process that nicely approximates the equilibrium behavior. This approximation leads him to introduce a new notion of evolutionary stability, local probabilistic stability (LPS), which requires that a population which begins play in equilibrium settle into a fixed stochastic pattern around the equilibrium. Bill shows that LPS is an appealing way to understand long run behavior in population models, in particular that it provides a criterion for understanding what sorts of long run behavioral patterns may be expected to emerge. In a series of subsequent papers, including papers in Games and Economic Behavior in 2007 ("Simple Formulas for Stationary Distributions and Stochastically Stable States" ) and two 2009 Journal of Economic Theory papers ("Stable Games and their Dynamics" and "Large-Population Potential Games"), Bill brings these techniques to fruition by providing complete analyses of important classes of problems. The concept of LPS has become a standard tool in evolutionary game theory.

Much of Bill's more recent work was concerned with exploring further the connections between individual behavior and population dynamics. Throughout this work, Bill exhibited his talent for constructive abstraction, isolating the essence of an idea by stripping away confounding detail. A number of Bill's early papers exploited the fact that if a population is large, then aggregate behavior will be almost deterministic, even if individual behavior is stochastic. In "Stochastic Approximations with Constant Step Size and Differential Inclusions" (SIAM Journal on Control and Optimization, 2013), a technically impressive paper, he extends existing approximation results, making precise the sense in which deterministic dynamics can be useful in characterizing the random behavior of a population of agents. "Sampling Best Response Dynamics and Deterministic Equilibrium Selection" ( Theoretical Economics, 2015), building on a 2001 IJGT paper, takes these ideas in a more applied direction, showing that if members of a population choose strategies by first observing the behavior of a few others in the population, and then playing a best response to this sample, then the resulting population behavior can be described by a deterministic system that selects so-called $p$-dominant equilibria. Models that link stochastic individual strategy choice to the aggregate selection of risk-dominant equilibria are familiar, but are often viewed as generating problematically long waiting times. Bill's paper is striking for the fact that it does not rely on ever-longer waiting times.

The behavior of evolutionary processes built up from stochastic individual behavior often hinges on "large deviations", or rare but critical bursts of behavior. Results from large deviation theory again appear in Bill's work, stretching from his 2010 paper in Theoretical Economics to his 2018 paper in Mathematics of Operations Research. In "Large Deviations and Stochastic Stability in the Small Noise Double Limit" (Theoretical Economics, 2016), Bill identifies conditions under which large deviations can be analyzed within optimal control theory. His forthcoming Mathematics of Operations Research paper, unfortunately to appear posthumously, extends this idea, again bringing impressive technique to bear.

There are yet more of Bill's papers that warrant attention. Evident throughout his work was a sense of curiosity. Bill was a leader in extending the purview of evolutionary game theory from random matching games in fixed populations to the study 
of quite general population games. While many others were content to consider the replicator dynamic, Bill developed and examined a host of alternatives, with "Riemannian" dynamics (Journal of Economic Theory, 2018) being one of his recent contributions. While many others were content to focus on best response dynamics, Bill again considered alternatives, with "best experienced payoff dynamics" (Journal of Economic Theory, 2020) being the most recent.

Beyond his research, Bill will be remembered for his collegiality, generosity, and humility. He was kind and generous, just as eager to talk about, or work on, others' research as his own. He was always more concerned with finding the truth than with worrying about getting credit for having found it. He was devoted to his students, with whom he was invariably gentle and encouraging, while insisting on exacting standards. He wrote innovative software for evolutionary dynamics, and then not only made it freely available but patiently answered questions from those seeking help with it. He put enormous energy into his teaching, drawing rave reviews for his undergraduate statistics course, otherwise usually viewed as the epitome of drudgery, and that teaching culminated in his recent Vital Statistics textbook. Bill made any group or project he was a part of work better. We miss him a lot.

Books

Population Games and Evolutionary Dynamics. MIT Press, 2010.

Vital Statistics: Probability and Statistics for Economics and Business (with Brett A. Saraniti). Oxford University Press, 2019.

\section{Software}

Abed: Agent-Based Evolutionary Dynamics (with Luis Izquierdo and Segis Izquierdo). luis-r-izquierdo.github.io/abed.

Dynamo: Phase Diagrams for Evolutionary Game Dynamics (with Emin Dokumaci and Francisco Franchetti). www.ssc.wisc.edu/ whs/dynamo.

EvoDyn-3s (with Luis Izquierdo and Segis Izquierdo). luis-r-izquierdo.github.io/ EvoDyn-3s.

Articles in refereed journals

"Hamilton-Jacobi Equations with State Constraints and Optimal Transition Paths for Stochastic Stability" (with Hung Tran and Srinivas Arigapudi). Mathematics of Operations Research, forthcoming

"Stability for Best Experienced Payoff Dynamics" (with Luis R. Izquierdo and Segis S. Izquierdo). Journal of Economic Theory 185 (2020)

"Best Experienced Payoff Dynamics and Cooperation in the Centipede Game" (with Segis Izquierdo and Luis Izquierdo). Theoretical Economics 14 (2019), 134-1385

"An Introduction to $A B E D$ : Agent-Based Simulation of Evolutionary Game Dynamics" (with Luis Izquierdo and Segis Izquierdo). Games and Economic Behavior 118 (2019), 434-462. 
"EvoDyn-3s: A Mathematica Computable Document to Analyze Evolutionary Dynamics in 3-Strategy Games (with Luis Izquierdo and Segis Izquierdo). SoftwareX 7 (2018), 226-233.

"Riemannian Game Dynamics" (with Panayotis Mertikopoulos). Journal of Economic Theory 177 (2018), 315-364.

"Sample Path Large Deviations for Stochastic Evolutionary Game Dynamics" (with Mathias Staudigl). Mathematics of Operations Research 43 (2018), 13481377.

"Learning in Games via Reinforcement and Regularization" (with Panayotis Mertikopoulos). Mathematics of Operations Research 41 (2016), 1297-1324.

"Large Deviations and Stochastic Stability in the Small Noise Double Limit" (with Mathias Staudigl). Theoretical Economics 11 (2016), 279-355.

"Sampling Best Response Dynamics and Deterministic Equilibrium Selection" (with Daisuke Oyama and Olivier Tercieux). Theoretical Economics 10 (2015), 243-281.

"Local Stability of Strict Equilibria under Evolutionary Game Dynamics." Journal of Dynamics and Games 1 (2014), 485-495.

"Probabilistic Interpretations of Integrability for Game Dynamics." Dynamic Games and Applications 4 (2014), 95-106.

"An Introduction to Dynamo: Diagrams for Evolutionary Game Dynamics" (with Francisco Franchetti). Biological Theory 8 (2013), 167-178.

"Stochastic Approximations with Constant Step Size and Differential Inclusions" (with Grégory Roth). SIAM Journal on Control and Optimization 51 (2013), $525-555$.

"Stochastic Imitative Game Dynamics with Committed Agents." Journal of Economic Theory 147 (2012), 2056-2071.

"Preface: Second DGAA Special Issue on Evolutionary Games" (with Ross Cressman and Christine Taylor). Dynamic Games and Applications 2 (2012), 1-3 "Large Deviations and Multinomial Probit Choice" (with Emin Dokumaci). Journal of Economic Theory 146 (2011), 2151-2158

"Preface: DGAA Special Issue on Evolutionary Games" (with Ross Cressman and Christine Taylor). Dynamic Games and Applications 1 (2011), 351-353

"Survival of Dominated Strategies under Evolutionary Dynamics" (with Josef Hofbauer). Theoretical Economics 6 (2011), 341-377.

"Decompositions and Potentials for Normal Form Games." Games and Economic Behavior 70 (2010), 446-456

"Local Stability under Evolutionary Game Dynamics." Theoretical Economics 5 (2010), 27-50.

"Orders of Limits for Stationary Distributions, Stochastic Dominance, and Stochastic Stability." Theoretical Economics 5 (2010), 1-26.

"Pairwise Comparison Dynamics and Evolutionary Foundations for Nash Equilibrium." Games 1 (2010), 3-17.

"Large Population Potential Games." Journal of Economic Theory 144 (2009), 1710-1725. "Stable Games and their Dynamics" (with Josef Hofbauer). Journal of Economic Theory 144 (2009), 1665-1693 
"Cultural Integration and Its Discontents" (with Timur Kuran). Review of Economic Studies 75 (2008), 201-228.

"The Projection Dynamic and the Geometry of Population Games" (with Ratul Lahkar). Games and Economic Behavior 64 (2008), 565-590.

"The Projection Dynamic and the Replicator Dynamic" (with Emin Dokumaci and Ratul Lahkar). Games and Economic Behavior 64 (2008), 666-683.

"Robust Permanence and Impermanence for Stochastic Replicator Dynamics" (with Michel Benaïm and Josef Hofbauer). Journal of Biological Dynamics 2 (2008), 180-195.

"Evolution in Bayesian Games II: Stability of Purified Equilibrium." Journal of Economic Theory 136 (2007), 641-667.

"Simple Formulas for Stationary Distributions and Stochastically Stable States." Games and Economic Behavior 59 (2007), 154-162.

"Evolution in Games with Randomly Disturbed Payoffs" (with Josef Hofbauer). Journal of Economic Theory 132 (2007), 47-69

"Pigouvian Pricing and Stochastic Evolutionary Implementation." Journal of Economic Theory 132 (2007), 367-382.

"Excess Payoff Dynamics and Other Well-Behaved Evolutionary Dynamics." Journal of Economic Theory 124 (2005), 149-170.

"Evolution in Bayesian Games I: Theory" (with Jeffrey C. Ely). Games and Economic Behavior 53 (2005), 83-109.

"Negative Externalities and Evolutionary Implementation." Review of Economic Studies 72 (2005), 885-915.

"Evolution and Equilibrium under Inexact Information." Games and Economic Behavior 44 (2003), 343-378.

"On the Global Convergence of Stochastic Fictitious Play" (with Josef Hofbauer). Econometrica 70 (2002), 2265-2294.

"Evolutionary Implementation and Congestion Pricing." Review of Economic Studies 69 (2002), 667-689

"Almost Global Convergence to $p$-Dominant Equilibrium." International Journal of Game Theory 30 (2001), 107-116.

"Preference Evolution, Two-Speed Dynamics, and Rapid Social Change." Review of Economic Dynamics 4 (2001), 637-679

"Potential Games with Continuous Player Sets." Journal of Economic Theory 97 (2001), 81-108.

"Evolution, Population Growth, and History Dependence" (with Ady Pauzner), Games and Economic Behavior 22 (1998), 84-120.

"History Independent Prediction in Evolutionary Game Theory." Rationality and Society 10 (1998), 303-326.

"Simple and Clever Decision Rules in a Model of Evolution." Economics Letters 61 (1998), 165-170. 
Articles in edited volumes

"Population Games and Deterministic Evolutionary Dynamics." In H. P. Young and S. Zamir, eds., Handbook of Game Theory, Vol. 4, 703-775. North Holland, 2015.

"Stochastic Evolutionary Game Dynamics: Foundations, Deterministic Approximation, and Equilibrium Selection." In K. Sigmund, ed., Evolutionary Game Dynamics, 111-141. American Mathematical Society, 2011.

"Evolutionary Game Theory." In R. A. Meyers, ed., The Encyclopedia of Complexity and Systems Science, 3176-3205. Springer, 2009. (Reprinted in R. A. Meyers, ed., Computational Complexity: Theory, Techniques, and Applications, 1000-1029. Springer, 2012.)

"Deterministic Evolutionary Dynamics." In S.N. Durlauf and L. E. Blume, eds., The New Palgrave Dictionary of Economics, 2nd edition, vol. 2, 457-461. Macmillan, 2008.

"Learning and Evolution in Games: Overview". In S. N. Durlauf and L. E. Blume, eds., The New Palgrave Dictionary of Economics, 2nd edition, vol. 5, 56-60. Macmillan, 2008.

Book reviews

"Strategic Learning and its Limits by H. Peyton Young." Games and Economic Behavior 63 (2008), 417-420.

"Evolutionary Dynamics and Extensive Form Games by Ross Cressman." International Review of Economics and Finance 15 (2006), 136-140.

Corr. Author for internal communication:

Romina Goldman

E-Mail: rominag@savion.huji.ac.il

Publisher's Note Springer Nature remains neutral with regard to jurisdictional claims in published maps and institutional affiliations. 